\title{
Current Treatment and Controversy of Primary Gastric Lymphoma*
}

\author{
Ming-Chih Chang \\ Mackay Memorial Hospital, Mackay Medicine, Nursing and Management College, Taipei, Chinese Taipei. \\ Email: mmhdonald@yahoo.com.tw
}

Received November $30^{\text {th }}$, 2012; revised December $29^{\text {th }}$, 2012; accepted January $9^{\text {th }}, 2013$

\begin{abstract}
Primary gastric lymphoma (PGL) is not a common cancer and account for 10\% of malignant lymphoma and 5\% of gastric cancer. The correlation with Helicobacter pylori (H. pylori) infection with mucosa associated lymphoepithelial tumor (MALT) is now well documented and some of the low grade MALT can be cured sorely by triple agent eradication therapy. The most common type of PGL is diffuse large B cell lymphoma which now can be successfully treated with chemotherapy alone. There is still no consensus on the optimal treatment for PGL. In the recent 10 years chemotherapy combined with anti-CD20 monoclonal antibody such as rituximab, achieved higher complete response rate and more than $80 \%$ are long-term survival. The so-called R-CHOP (rituximab, cyclophosphamide, vincristin, prednisolone) now become the new gold standard therapy. The role of surgical resection prior to chemotherapy is controversial and not commonly applied in recent publications. Yet some cases of suboptimal response to R-CHOP or patient is too fragile to tolerate the immuno-chemotherapy will be feasible to surgical resection as a salvage or alternative therapy. The radiotherapy as an adjuvant therapy is less commonly considered. Patients with advanced PGL with high international prognostic index risk and along with co-morbidity diseases are prone to get treatment related complications from abovementioned modality of treatment, such as GI perforation, neutropenic septicemia, pulmonary infection, fulminate heaptitis $\mathrm{B}$ reactivation, respiratory and cardiac impairment can be seen.
\end{abstract}

Keywords: Primary Gastric Lymphoma; MALT Lymphoma; Combination Rituximab and Chemotherapy; Surgery

\section{Introduction}

With great improvement of modern chemotherapy and supportive care, consequently a global therapeutic approach to the cure of PGL has completely changed over the last one decade: innovative, conservative options to reduce treatment toxicity, thus preventing systemic relapses, have made their appearance and are on the rise. In the recent 10 years chemotherapy combined with antiCD20 monoclonal antibody such as rituximab, achieved higher complete response rate and more than $80 \%$ are long-term survival [1]. The role of surgical resection prior to chemotherapy is controversial and not commonly applied in recent publications [1-4]. Among the gastric malignancies PGL has the most favorable outcome not only in localized disease but also in stage IIb/IV disease. It is highly sensitive to chemotherapy and anti-CD20 immunotherapy [1-3]. The primary role of surgery or chemotherapy remains the mainstay is an issue of debate [4]. However, there is, as yet, no randomized control trial to define the primary treatment of PGL, either surgery or

\footnotetext{
*The author declares of no conflict of interest with regarding industrial companies.
}

chemotherapy or combined modality. This article tries to answer this controversy and also delineate the current treatment of MALT lymphoma. Is it not yet clear that $H$. pylori eradication alone can cure the gastric MALT? And use chemotherapy is justified afterward for partial tumor regression or till disease progression. Also the pathogenesis and genetic aberration of large cell transformation from high grade MALT will be discussed.

\section{Pathogenesis of Gastric Lymphoma and MALT}

In histology all of the PGL originated from B lymphocyte, the normal counterpart is germinal center B cell. The cause of PGL is partly from de novo DLBCL, and partly transformed from low to high grade MALT and lastly diffuse large B-cell lymphoma (DLBCL). The cytokine changes and genetic aberration give this pathogenesis. Issacson et al. proposed a theory that the early MALT caused by chronic inflammation triggered by infection or autoimmune disorders, including $H$. pylori gastritis, Sjögren syndrome [5]. It causes abnormal B cell proliferation and T-cell infiltration around the MALT. 
Recently two recurrent chromosomal changes in this tumor were identified-the $t(11 ; 18)(q 21 ; q 21)$ and the $t(1 ; 14)$ (p2;q32). The $t(11 ; 18)$ produce the fusion of AIP2 at 11q21which encode of inhibitor of apoptosis protein IAP to a gene at 18q21 named MLT1. The segment of MLT1 bind to BCL10, this fusion protein strongly activate NF-kB, in which thus suppress host cell apoptotic response to viral infection [6]. Once the fusion gene developed the gastric MALT become $H$. pylori independent growth, and no longer regress by antibiotics eradication.

\section{Histological Classification of PGL}

Primary malignant lymphoma of the stomach are almost all non-Hodgkin's type and of B-cell lineage. These lymphomas usually arise from MALT, also known as marginal zone B-cell lymphoma (Low and High grade). Diffuse large B-cell lymphoma is most common, including high grade lymphoma of MALT origin with large cell transformation and non-MALT type and they are indistinguishable. Other types include mantle cell lymphoma (malignant lymphomatous polyposis), follicular lymphoma, Burkitt or T-cell lymphoma are quite rare, often involving intestinal tract as well and may not be sole gastric lesion. The diagnosis is made on the morphological features. Lymphoma should not be diagnosed on the basis of clonality studies by immunohistochemistry or molecular techniques alone. It commonly located in the gastric antrum. It may be polypoid and fungating like lesion mimicking gastric carcinoma. In low grade cases, multiple erosions or superficial ulceration may be present. Lymphoepithelial lesions defined as glandular structures expanded and destroyed by groups of more than 3 lymphoid cells. Immunostaining for CD20, CD79 and cytokeratin are useful for demonstrating lymphoepithelial lesions. CD5, CD10, CD23 or cyclin D1 are negative [5-7]. Occasional non destructive lymphoepithelial lesions alone are not sufficient to diagnose lymphoma. Carefully look for compact clusters, confluent aggregates or sheets of blast cells that makes the tumor high grade. The high grade MALT is characterized by destructive infiltrate of clusters or sheets of blast cells with few or no lymphoepithelial lesions. Mitoses and apoptotic bodies frequent. It may be difficult in distinguishing from diffuse carcinoma, sarcoma, or even T-cell lymphoma or metastatic melanoma [7]. Epithelial lesions infiltrated by melanoma cells may mimic lymphoepithelial lesions. Mucin stains and immunostaining for cytokeratin, CEA, common leukocyte antigen, B- and Tcell markers are very helpful. Some MALT lymphomas have been reported in immunocompromised patients.

\section{Clinical Features of PGL}

The most common symptom at presentation is abdominal pain account for $80 \%$ followed by melena $36 \%$, nausea, vomiting, hematemesis and ileus. B-symptoms as fever, body weight loss are common, and some bulky disease. Most of PGL located at antrum followed by corpus and fundus [8]. The gastrosopic findings varied from bulging mass, ulcerative lesion, submucosal tumor. The median age is around 50 to 55 years, male to female ratio 1.2:1. Increase LDH serum level is common, even for localized lymphoma [8]. The ratio of GCB (germinal center B cell, less aggressive) to non-GCB phenotypes was about 1:1.85. MALT lymphoma accounted for $20 \%-40 \%$ varied from series [9].

\section{Staging of PGL}

Classification of the tumor stage was according to the Musshoff's modification of the Ann Arber staging system [10]. Stage IE denotes tumor remains confined within the stomach; stage IIE-1, which has perigastric nodal involvement only, and stage IIE-2, which has more distant nodal involvement below the diaphragm. Stage IIIE denotes nodal involvement above and below the diaphragm, and stage IV denotes multiple visceral organ involvement. All tumors were evaluated abdominal/chest CT scan and endoscopy or endoscopic ultrasound. Lugano staging system was also applied as well and it omit the stage III disease [11].

\section{Management of PGL}

\subsection{Role of Surgery}

Traditionally the gastric lymphoma was managed by surgeon with partial or total gastrectomy not only for diagnosis, staging and for cure purpose. Yet there were substantial patients relapsed and adjuvant chemotherapy after surgery was considered. In deed, to the best of our knowledge, from a thorough review of the literature, most of the relevant literature pertaining to this surgery has related to studies featuring, generally, small numbers of patients as well as such studies having been conducted retrospectively and during the 1980s and the 1990s. Thus, many investigators still advocate surgical resection in order to debulk the lesion mass and to accurately stage the lesion to attempt to prevent post-surgical perforation [12-15]. During the early 1990s, some authors had suggested that chemotherapy alone was effective for the sole management therapy modality and may also have been sufficiently effective to prevent the morbidity associated with gastrectomy, such a conservative approach supporting gastric preservation for subsequent residual diseases [16-19]. Further, yet other authors have recommended surgery as being able to affect a cure for primary gastric lymphoma, particularly for stage IE disease [2022]. Those researchers who suggested surgery either with 
or without associated chemotherapy focused on the accurate staging of the tumor, less hemorrhage or perforation of the stomach during the treatment; although subsequent to the development of endoscopic ultrasound, the role of laparotomy biopsy has, ultimately, become unwarranted [20]. Meanwhile, most of the reports have revealed a rather low incidence of severe hemorrhage or perforation, accounting for, respectively, $2.1 \%$ and $1.7 \%$ of those individuals treated with chemotherapy without surgery and, respectively, $2.2 \%$ and $0.9 \%$ of those individuals treated only surgically $[23,24]$. Such evidence does suggest that the role of surgery for treatment of primary gastric lymphoma may be less important than previously considered.

\subsubsection{The Controversies of Primary Treatment of PGL}

The issue of optimal treatment for localized PGL has not been resolved. In the 1980s, gastrectomy, because of its low surgery-related mortality ( $2 \%-5 \%)$, was used to treat PGL [20-23]. According to results from the Mayo Clinic, the 5-year-survival rate was reported to reach $75 \%$ with curative resection, and 32\% with palliative resection [14]. For stage IE PGL, the cure rate could be even as high as $80 \%$. However, the success of surgical management of PGL depends on the size of tumor, the depth of tumor penetration into gastric tissue, and the involvement of regional lymph nodes [20]. Some investigators began using chemotherapy, mostly CHOP [12,14, 23] and R-CHOP after year 2000 [1-3], to control the tumors and to prevent postoperative morbidity. A study by Maor et al. showed that the six-year overall survival of patients treated with chemotherapy alone was $76 \%$ [14]. The recent publications gave even better results with 5 -year OS over 90\% [1,2]. However, for bulky tumors, the advantage of chemotherapy is offset by the potential tumor bleeding and gastric perforation [4]. In 1999 there was a publication of international survey to investigate the treatment consenus [24]. It reviewed the management of gastric lymphoma, formatted questionnaires were mailed to leading institutes with a special interest in this field in 19 centers of Europe, the United States and Japan. Gastroenterologists are far more inclined to perform a (partial) gastric resection (with or without additional radiation therapy or chemotherapy) than haematologists and medical oncologists. And some European center as well as USA preferred to use radiotherapy as adjuvant or salvage therapy [24,25]. Therefore, some investigators suggested that debulking surgery followed by chemotherapy and/or radiotherapy, so-called combined modality, achieved better clinical outcome mainly better tumor control with reduced complication rates [26-32]. In our series, all five patients who developed gastric perforation were in the chemotherapy-alone group, and eventually died of this complication. In contrast, none of the patients receiving the combination therapy had this complication, suggesting an important role for surgery in selected patients [4]. Of note, five patients of major complication, four in the chemotherapyalone group and one in the combination therapy group, developed gastro-intestinal bleeding at the time of disease progression. The overall surgical morbidity and mortality were $15.4 \%(2 / 13)$ and $7.7 \%$ (1/13), respectively, similar to what has been reported by Rackner et al. [33]. The rate of surgical complications might offset the benefit expected from tumor control. This notion is further supported by the results of Salles et al., showing that for localized PGL, surgical resection prior to chemotherapy did not affect the complete response rate, survival rate, or the disease-free survival [12]. Similar results have also been demonstrated in several studies on Asian patients and in the Groupe d'Etude des Lymphomes Digestifs (GELD) and Groupe d'Etude des Lymphomes de l'Adulte (GELA) studies [34-39]. Such evidence suggests the role of surgery in the primary treatment of PGL is declined.

Although surgery for PGL appears to have a diminished role in recent studies, it might enhance the effects of chemotherapy in stage IE PGL $[40,41]$ Patients treated with conservative surgery followed by three cycles of chemotherapy had a better five-year survival rate than patients treated with chemotherapy alone [26,30-32]. We also noticed an improved outcome in patients who received treatment after year 2000, probably due to better supportive care and a more widespread use of G-CSF for febrile neutropenia. A subgroup of patients was identified who might be more likely to develop chemotherapy-induced life-threatening complications. Often treated in an outpatient setting, these patients failed to achieve a complete response to chemotherapy. To avoid such severe complications, we recommend re-evaluating patients by endoscopy after two cycles of chemotherapy. At the same time, patients should be warned that complications such as gastric perforation and bleeding, although not common, are possible and be made more aware through comprehensive education.

In real world practice, it would appear that the gastroenterologist's point of view would be crucial as regards the making of a decision in algorithmic approach, in which whether to send patients to a medical oncologist or a surgeon [24]. Although chemotherapy alone is very effective in terms of complete response rate and 5-year disease free survival and overall survival comparable to surgery plus adjuvant chemotherapy [1-4]. Yet, not all the gastroenterologist follow this recommendation, and used to referring patients to medical oncologist and surgeon at the same time; more or less they prevail the surgery role and also notice the importance of chemotherapy 
to circumvent distant metastasis.

In one report from China, 200 cases of PGL, 24 cases received chemotherapy alone, 29 cases surgery alone, 132 cases underwent surgery plus chemotherapy, and 15 were of palliation [42]. The ten-year survival rates were $55.0 \%$. Another study identified 79 patients 30 patients (38\%) underwent surgery, 74 (92\%) received chemotherapy, and 18 (23\%) received radiotherapy. The fiveyear OS and DFS rates were $91.2 \%$ and $83.9 \%$, respectively, in patients with stage I/II or IIE disease and $70.6 \%$ and $65.5 \%$, respectively. Treatment modality (surgical or conservative) had no impact on OS or DFS in early stages [43].

\subsubsection{The Role of Rituximab Containing Chemotherapy}

The safety of chemotherapy is improving with modern supportive care, giving more survival benefit. The emergence of anti-CD 20 monoclonal antibody such as Rituximab proved to be very effective in treating B-cell lymphoma. The GELA study showed a $15 \%$ ten-yr disease free survival benefit, comparing R-CHOP with CHOP, in overall diffuse large B cell lymphoma, but no data specialized for PGL. The PGL treatment make a new paradigm, R-CHOP is a front line treatment of choice. We will recommend CHOP-21 for six cycles and rituximab for 8 cycles, which is R-CHOP-21 for six cycles followed by rituximab for 2 more cycles.

One study from Japan evaluates clinical outcomes of PGL in the rituximab era, they conducted a retrospective, multicenter analysis of 95 patients with PGL. In 58 patients with localized disease, 3-year progression-free survival (PFS) and overall survival (OS) were 91\% and 91\% for patients with six cycles of rituximab plus CHOP (R-CHOP) and 92\% and 95\% for patients with three to four cycles of R-CHOP plus radiotherapy (Log-rank test, $\mathrm{P}=0.595$ and $\mathrm{P}=0.278$, respectively). No patient underwent surgery [1]. Clinical outcomes of PGL were extremely favorable and promising for localized-stage patients in the rituximab era [3]. Also recent report of Chinese patients who received chemotherapy with rituximab (at least 3 cycles) had a mean OS of 72 months (95\% CI 62 - 81) versus 62 months (95\% CI 47 - 76) for patients chemotherapy alone $(\mathrm{P}=0.021)$. This study reflected the additive benefit of rituximab when combined with chemotherapy [42]. However, a phase II clinical trial showed that the addition of rituximab to standard chemotherapy did not improve the outcome in early-stage PGL due to high long-term survival in both groups [2].

\subsubsection{The Issues of Treatment Related Complications}

In those patients who achieved the complete response, the very late relapse of gastric lymphoma is quite rare and still can be re-treated with same chemotherapy or salvage chemotherapy. The toxicity and safety for $\mathrm{R}-\mathrm{CHOP}$ is an important issue. Some patients is rather fragile especially for the elderly, the drawback of R-CHOP regimen will give $10 \%$ - $20 \%$ grade III/IV febrile neutropenia. The infection rate increase in dose-density regimen. The most troublesome side effects of R-CHOP are pulmonary infection such as PJP pneumonia, pulmonary interstitial disease, cardiovascular event, congestive heart failure, and reactivation of hepatitis B in East Asia countries. The fulminant hepatitis account for $10 \%-16 \%$ in hepatic B antigen positive carrier without anti-viral prophylaxis, and some of them succumb to death due to hepatic failure. The hepatitis B seroconvertsion from anti-HBc to HBsAg positive rate is around 6\% - $8 \%$, and very low death rate, partly due to early recognition and low HBV-DNA viral load. We recommend early anti-viral prophylaxis by using lamivudine or entercavir even though the liver function is normal. This prophylaxis should be used for 12 months or 6 months after completion of chemotherapy. The interstitial lung fibrosis and anthracycline or rituximab related cardiomyopathy is another important issue, which needs high clinical alert and pay much attention to patients with pre-existing co-morbidity. R-CVP will be an alternative regimen for fragile patients.

\subsubsection{Advanced PGL}

Advanced PGL is still a challenging issue and the improvement of modern treatment is not satisfactory. A series of 37 patients with advanced disease, 3-year PFS and 3-year OS were $43 \%$ and $64 \%$ for patients with R-CHOP chemotherapy with or without radiotherapy [1]. The prognosis is still poor for advanced-stage patients even in the rituximab era. In our series, the overall survival was significantly lower for advanced PGL patients than localized PGL patients, signifying again that stage is an important prognostic factor in the treatment of PGL [4]. In the chemotherapy-only group, three out of seven complete responders of advanced PGL patients (3/19 or $15.8 \%$ ) were long-term survivors (DFS more than 6 years), while all four patients in the combined-modality group died. The outcome of combined-modality group is dismal due to higher incidence of treatment related tumor bleeding as well as febrile neutropenia, the incidence was $75.0 \%$ and $50 \%$ respectively, than chemotherapy alone group, $31.6 \%$ and $15.8 \%$, respectively. The role of surgery is limited in the treatment of advanced PGL in our series. How to conduct effective high dose chemotherapy for advanced PGL and counterbalance the severe adverse event is the current issue to be answered.

\section{Treatment of MALT Lymphoma}

The MALT lymphoma account for $40 \%$ - 45\% all gastric 
lymphoma, it can be classified as low grade MALT, high grade MALT and transformed from high grade to DLBCL. The low grade MALT is very indolent and always almost localized disease. Patients shall receive a triple therapy with omeprazole (20 $\mathrm{mg}$ twice a day), amoxicillin (1 g twice a day) and clarithromycin (500 mg twice a day) for 14 days, followed by omeprazole for other 21 days. One report of 61 patients with primary gastric large B-cell lymphoma were treated with anti $H P$ treatment and 42 of them showed a complete response [45]. The median time to complete response was 9.6 months for MALT with high grade predominant and 5.5 months for low grade MALT. The longer period of follow-up was reported in this study showed all the 14 MALT patients with CR remained relapse-free after a median follow-up of 63 months [46]. Histological regression of the lymphoma was complete in 19/44 patients (43\%). No regression was noted in the $10 \mathrm{H}$. pylori negative patients. Among the $34 \mathrm{H}$. pylori positive patients, the $H$. pylori eradication rate was $100 \%$; complete regression rate of the lymphoma increased from 56\% $(19 / 34)$ to $79 \%(19 / 24)$ when there was no nodal involvement at endoscopic ultrasonography [47]. There was a significant difference between the response of the lymphoma restricted to the mucosa and other more deep seated lesions $(P<0.006)$. However, using multivariate analysis, the only predictive factor of regression was the absence of nodal involvement $(\mathrm{P}<0.0001)$ [48].

The high grade MALT can be either treated with anti- $H$. pylori regimen alone or combined with short course of CHOP or R-CHOP. Some authors advocate anti- $H$. pylori regimen alone will be sufficient to cure the disease, however we may overlooked the potential sampling error that some part of MALT is undergoing transformation to DLBCL, which is already become autonomy and independent from MALT1 gene control. In our clinical observation there is a part of high grade MALT is not cured by anti-H. pylori regimen alone and turned to be wide-disseminated MALT disease. The risk factors will be MALT infiltration to muscularis mucosa, nodal involvement and presence of $\mathrm{t}(11 ; 18)(\mathrm{q} 21 ; 21)$. It needs not to be transformed as DLBC before dissemination, and keeps the original morphology as extranodal marginal zone lymphoma. It is not uncommon to see patients histology presented with composite high grade MALT and DLBCL in same specimen. We do not routinely used chemotherapy for high grade MALT, but staging procedure should be done as an indolent or aggressive lymphoma. The stage III/IV disease should be treated as advanced indolent lymphoma accordingly. The MALT can be disseminated or present at an advanced stage or involved other extranodal sites as approximately one third of cases [49]. For those patients of high grade MALT with transformation and composite with DLBCL, the recommendation will be anti-H. pylori regimen followed by four cycles with CHOP or R-CHOP; whereas anti- $H$. pylori regimen followed by four to six cycles of rituximab remained for the elderly and fragility.

\section{Discussion}

The global therapeutic approach to the cure of PGL has completely changed over the past one decade with great success by improvement of modern chemotherapy and supportive care. The chemotherapy combined with antiCD 20 monoclonal antibody such as rituximab, achieved higher complete response rate and more than $80 \%$ are long-term survival. Regarding with the treatment modality, we'd better keep open-minded approach. In our own series, we refer patients to surgeon for subtotal gastrictomy for those with large tumor and high co-morbidity, and preserve radiation therapy for those who are not feasible for operation in what manner. And add two to four cycles of post-operative salvage chemotherapy as consolidation purpose. Subtotal gastrectomy is justified in selected cases. Surgery is indicated to treat complications such as gastric perforation, uncontrolled hemorrhage. A small portion of patients may encounter small amount melena passage most often one week after chemotherapy due to tumor necrosis, which happened during first two cycles. It always a transient course and resolved with supportive care.

In real world practice, after gastroscopic biopsy proved PGL, we undergo staging procedure, risk stratification, and chemotherapy is the primary treatment given to patients without surgery. The local control and overall survival is not different from surgery. The toxic death rate is negligible. For those centers where surgery is the standard primary treatment, subtotal gastrectomy replaces total gastrectomy, yet adjuvant chemotherapy should be done for those patients with positive surgical margin, large tumor $(>5 \mathrm{~cm})$ and lymph nodes involvement.

Those researchers who advocated surgery either with or without chemotherapy focused on the accurate staging of the tumor and the association of this treatment with less hemorrhage or perforation of the stomach; although with the advent of endoscopic ultrasound, laparotomy biopsy is no longer warranted [8]. We also consider surgery alone will be sufficient to cure PGL in low risk, stage IE disease, and it will be a good alternative primary treatment for those patients who are fragile and prone to develop R-CHOP related afore-mentioned complications.

\section{Conclusion and Future Perspectives}

The treatment paradigm of PGL is shifting from surgery to combined surgery and chemotherapy or radiotherapy, then to chemotherapy alone and finally rituximab containing chemotherapy is considered as the mainstay. The 
role of surgery decline due to very effective medical treatment, and R-CHOP alone achieve very high response rate and long term survival. In fact, it overshadows the primary role of surgery in treating either localized or advanced PGL. However, surgery is still justified in selected patients who may not tolerate immuno-chemotherapy or related complications. The outcome of advanced PGL and refractory/relapsed PGL is still dismal. The salvage chemotherapy can be used but with no clinical evidence yet, the morbidity will be substantial in high dose density regimen including auto or allo-stem cell transplant. Recently an old drug but newly indicated in malignant lymphoma is bendamustine, a cytotoxic drug which has both chemical structure of cyclophosphamide and fludarabin-like purine analogue, giving less myelosupression and cardiac toxicity [50]. The combination of rituximab and bendamustine regimen currently indicated in chronic lymphocytic lymphoma, follicular lymphoma and DLBCL as well [51]. Also the unraveling gene signature of DLBCL, the bortezomib and lenalidomide made BCL-2 and c-Myc driven pathogenesis the susceptible targets $[52,53]$. With those new and better armamentariums, the path of cure of PGL is promising and solid.

\section{Acknowledgement}

The author would like to acknowledge Dr Shi-Chuan Lin, Dr Li-Chong Chen for their review and advice to this article, and Dr Yun-Liang Chang for the preparation of this manuscript.

\section{REFERENCES}

[1] T. Tanaka, K. Shimada, K. Yamamoto, Y. Hirooka, et al., "Retrospective Analysis of Primary Gastric Diffuse Large B Cell Lymphoma in the Rituximab Era: A Multicenter Study of 95 Patients in Japan,” Annals of Hematology, Vol. 91, No. 3, 2012, pp. 383-390. doi:10.1007/s00277-011-1306-0

[2] A. Aviles, C. Castaneda, S. Cleto, N. Neri, et al., "Rituximab and Chemotherapy in Primary Gastric Lymphoma," Cancer Biotherapy \& Radiopharmaceuticals, Vol. 24, No. 1, 2009, pp. 25-28. doi:10.1089/cbr.2008.0507

[3] S. Wohrer, A. Puspok, J. Drach, M. Hejna, et al., "Rituximab, Cyclophosphamide, Doxorubicin, Vincristine and Prednisone (R-CHOP) for Treatment of Early-Stage Gastric Diffuse Large B-Cell Lymphoma," Annals of Oncology, Vol. 15, No. 7, 2004, pp. 1086-1090. doi:10.1093/annonc/mdh261

[4] M. C. Chang, M. J. Huang, Y. W. Su, et al., "Clinical Outcome of Primary Gastric Lymphoma Treated with Chemotherapy Alone or Surgery Followed by Chemotherapy," Journal of the Formosan Medical Association, Vol. 105, No. 3, 2006, pp. 194-202.

doi:10.1016/S0929-6646(09)60305-3
[5] P. G. Isaacson, “Gastrointestinal Lymphoma,” Human Pathology, Vol. 25, 1994, pp. 1020-1029. doi:10.1016/0046-8177(94)90060-4

[6] T. Yoshino, K. Omonishi, K. Kobayashi, T. Mannami, et al., "Clinicopathological Fearture of Mucosa Associated Lymphoid Tissue (MALT) Lymphoma: High Grade Transformation and Comparison with Diffuse Large B Cell Lymphoma without MALT Lymphoma Features," Journal of Clinical Pathology, Vol. 53, 2000, pp. 187-190. doi:10.1136/jcp.53.3.187

[7] E. S. Jaffe, N. L. Harris, H. Stein and J. W. Vardiman, "World Health Organization Classification of Tumours. Pathology and Genetics of Tumours of Haematopoietic and Lymphoid Tissues,” IARC Press, Lyon, Vol. 2001.

[8] F. d'Amore, H. Brinker, J. Gronbak, et al., "Non-Hodgkin's Lymphoma of the Gastrointestinal Tract: A Population-Based Analysis of Incidence, Geographic Distribution, Clinicopathological Presentation, Features, and Prognosis,” Journal of Clinical Oncology, Vol. 12, No. 8, 1994, pp. 1673-1684.

[9] J. Huang, W. Jiang, R. Xu, H. Huang, et al., "Primary Gastric Non-Hodgkin's Lymphoma in Chinese Patients: Clinical Characteristics and Prognostic Factors,” BMC Cancer, Vol. 10, 2012, p. 358. doi:10.1186/1471-2407-10-358

[10] K. Mushoff, "Klinische Stadieneineteilung der Nicht-Hodgkin's Lymphoma,” Strahlentherapie und Onkologie, Vol. 153, 1977, pp. 218-221.

[11] A. Rohatiner, F. d'Amore, B. Coiffier, et al., "Report on a Workshop Convened to Discuss the Pathological and Staging Classifications of Gastrointestinal Tract Lymphoma,” Annals of Oncology, Vol. 5, No. 5, 1994, pp. 397-400.

[12] G. Salles, R. Herbrecht, H. Tilly, et al., “Aggressive Primary Gastrointestinal Lymphomas: Review of 91 Patients Treated with the LNH-84 Regimen. A Study of the Group d'Etude des Lymphomes Agressifs," American Journal of Medicine, Vol. 90, No. 1, 1991, pp. 77-84. doi:10.1016/0002-9343(91)90509-V

[13] M. Raderer, J. Valencak, C. österreicher, et al., "Chemotherapy for the Treatment of Patients with Primary High Grade Gastric B-Cell Lymphoma of Modified Ann Arbor Stages IE and IIE,” Cancer, Vol. 88, No. 9, 2000, pp. 1979-1985.

doi:10.1002/(SICI)1097-0142(20000501)88:9<1979::AID -CNCR1>3.0.CO;2-L

[14] M. H. Maor, W. S. Valesquez, L. M. Fuller, et al., "Stomach Conservation in Stage IE and IIE Gastric NonHodgkin's Lymphoma,” Journal of Clinical Oncology, Vol. 8, No. 2, 1990, pp. 266-271.

[15] M. H. Shiu, L. Z. Nisce, A. Pinna, et al., "Recent Results of Multimodal Therapy of Gastric Lymphoma,” Cancer, Vol. 58, No. 7, 1986, pp. 1389-1399. doi:10.1002/1097-0142(19861001)58:7<1389::AID-CNC R2820580702>3.0.CO;2-Y

[16] F. A. Sheperd, W. K. Evans, G. Kutas, et al., "Chemotherapy Following Surgery for Stage IE and IIE NonHodgkin's Lymphoma of the Gastrointestinal Tract," Journal of Clinical Oncology, Vol. 6, No. 2, 1988, pp. 
253-260.

[17] M. K. Gospodarowicz, S. B. Sutcliffe, R. M. Clark, et al., "Outcome Analysis of Localized Gastrointestinal Lymphoma Treated with Surgery and Postoperative Irradiation," International Journal of Radiation Oncology, Biology and Physics, Vol. 19, No. 6, 1990, pp. 1351-1355. doi:10.1016/0360-3016(90)90343-I

[18] C. Rosen, J. A. Heerden, L. K. Martin, et al., "Is an Aggressive Surgical Approach to the Patient with Gastric Lymphoma Warranted?” Annals of Surgery, Vol. 205, No. 6, 1987, pp. 634-640. doi:10.1097/00000658-198706000-00004

[19] M. S. Hockey, J. Powel, J. Crocker, et al., "Primary Gastric Lymphoma,” British Journal of Surgery, Vol. 74, No. 6, 1987, pp. 483-487. doi:10.1002/bjs.1800740620

[20] R. J. Schwarz, J. M. Conners, N. Schmidt, et al., "Diagnosis and Management of Stage IE and Stage IIE Gastric Lymphomas," The American Journal of Surgery, Vol. 165, No. 5, 1993, pp. 561-565. doi:10.1016/S0002-9610(05)80435-0

[21] E. D. Durr, J. A. Bonner, J. G. Strickler, et al., "Management of Stage IE Primary Gastric Lymphoma," Acta Haematologica, Vol. 94, No. 2, 1995, pp. 59-68. doi:10.1159/000203975

[22] S. S. Yoon, D. G. Coit, C. S. Portlock, et al., "The Diminishing Role of Surgery in the Treatment of Gastric Lymphoma,” Annals of Surgery, Vol. 240, No. 2004, pp. 28-37. doi:10.1097/01.sla.0000129356.81281.0c

[23] A. Avilés, M. J. Nambo, N. Neri, et al., "The Role of Surgery in Primary Gastric Lymphoma: Results of a Controlled Clinical Trial,” Annals of Surgery, Vol. 240, No. 1, 2004, pp. 44-50. doi:10.1097/01.sla.0000129354.31318.f1

[24] D. de Jong, B. M. P. Aleman, B. G. Taal, et al., "Controversies and Consensus in the Diagnosis, Work-Up and Treatment of Gastric Lymphoma: An International Survey," Annals of Oncology, Vol. 10, No. 3, 1999, pp. 275280. doi:10.1023/A:1008392022152

[25] T. P. Miller, S. Dahlberg, J. R. Cassady, et al., "Chemotherapy Alone Compared Chemotherapy Plus Radiotherapy for Localized Intermediate- and High-Grade NonHodgkin's Lymphoma," The New England Journal of Medicine, Vol. 339, 1998, pp. 21-26. doi:10.1056/NEJM199807023390104

[26] W. P. Sheridan, G. Medley and G. N. Brodie, "NonHodgkin's Lymphoma of the Stomach: A Prospective Pilot Study of Surgery plus Chemotherapy in Early and Advanced Disease,” Journal of Clinical Oncology, Vol. 3, No. 4, 1985, pp. 495-500.

[27] F. A. Shepherd, W. K. Evans, G. Kutas, et al., "Chemotherapy Following Surgery for Stages IE and IIE NonHodgkin's Lymphoma of the Gastrointestinal Tract," Journal of Clinical Oncology, Vol. 6, No. 2, 1988, pp. 253-260.

[28] D. C. Cooper, R. Doria, E. Salloum, et al., "Primary Gastrointestinal Lymphomas,” Gastroenterologist, Vol. 4, 1996, pp. 54-64.

[29] A. Ruskoné-Fourmestraux, P. Aegerter, A. Delmer, et al.,
"Primary Digestive Tract Lymphoma: A Prospective Multicentric Study of 91 Patients-Group d'Etude des Lymphoma Digestifs,” Gastroenterology, Vol. 105, No. 6, 1993, pp. 1662-1671.

[30] P. G. Gobbi, P. Dionigi, F. Barbieri, et al., "The Role of Surgery in the Multimodal Treatment of Primary Gastric Non-Hodgkin's Lymphoma: A Report of 76 Cases and Review of the Literature," Cancer, Vol. 65, No. 11, 1990, pp. 2528-2536.

doi:10.1002/1097-0142(19900601)65:11<2528::AID-CN CR2820651123>3.0.CO;2-W

[31] G. Bellesi, A. Alterini, A. Messori, et al., "Combined Surgery and Chemotherapy for the Treatment of Primary Gastrointestinal Intermediate- or High-Grade Non-Hodgkin's Lymphomas,” British Journal of Cancer, Vol. 60, 1989, pp. 244-248. doi:10.1038/bjc.1989.262

[32] C. Tondini, F. Giardini, P. Bezztti, et al., "Combined Modality Treatment for Primary Gastrointestinal NonHodgkin's Lymphoma: The Milan Cancer Institute Experience,” Annals of Oncology, Vol. 4, No. 10, 1993, pp. 831-837.

[33] V. L. Rackner and J. A. Thirlby, "Ryan Role of Surgery in Multimodality Therapy for Gastrointestinal Lymphoma,” The American Journal of Surgery, Vol. 161, No. 5, 1991, pp. 570-575. doi:10.1016/0002-9610(91)90902-P

[34] A. Ruskoné-Fourmestraux, P. Aegerter, A. Delmer, et al., "Primary Digestive Tract Lymphoma: A Prospective Multicentric Study of 91 Patients-Group d'Etude des Lymphoma Digestifs,” Gastroenterology, Vol. 105, No. 6, 1993, pp. 1662-1671.

[35] H. T. Liu, C. Hsu, C. L. Chen, et al., "Chemotherapy Alone versus Surgery Followed by Chemotherapy for Stage I/IIE Large-Cell Lymphoma of the Stomach,” American Journal of Hematology, Vol. 64, No. 3, 2000, pp. 175-179.

doi:10.1002/1096-8652(200007)64:3<175::AID-AJH6>3. $\underline{0 . \mathrm{CO} ; 2-7}$

[36] B. Coiffier, C. Gisselbrecht, R. Herbrecht, et al., "LNH84 Regimen: A Multicentric Study of Intensive Chemotherapy in 737 Patients with Aggressive Malignant Lymphoma," Journal of Clinical Oncology, Vol. 7, 1989, pp. 1018-1026.

[37] S. Nakamura, T. Matsumoto, M. Iida, et al., "Primary Gastric Lymphoma in Japan: A Clinicopathologic Analysis of 455 Patients with Special Reference to Its Time Trends,” Cancer, Vol. 97, No. 10, 2003, pp. 2462-2473. doi:10.1002/cncr.11415

[38] C. L. Ho, A. T. Hsieh, M. S. Dai, et al., “Non-Hodgkin’s Lymphoma of the Stomach: Treatment Outcomes for 57 Patients over a 20-Year Period," Journal of the Chinese Medical Association, Vol. 68, No. 1, 2005, pp. 11-15. doi:10.1016/S1726-4901(09)70125-X

[39] M. Binn, A. Rouskoné-Fourmestraux, E. Lepage, et al., "Surgical Resection plus Chemotherapy versus Chemotherapy Alone: Comparison of Two Strategies to Treat Diffuses Large B-Cell Lymphoma,” Annals of Oncology, Vol. 14, No. 12, 2003, pp. 1751-1757. doi:10.1093/annonc/mdg495

[40] J. C. Vaillant, A. Ruskoné-Fourmestraux, P. Aegerter, et 
al., "Management and Long-Term Results of Surgery for Localized Gastric Lymphomas," The American Journal of Surgery, Vol. 179, No. 3, 2000, pp. 216-222. doi:10.1016/S0002-9610(00)00295-6

[41] B. P. Koch, F. del Valle, W. E. Berdel, et al., "Primary Gastrointestinal Non-Hodgkin's Lymphoma: II. Combined Surgical and Conservative or Conservative Management Only in Localized Gastric Lymphoma-Results of the Prospective German Multicenter Study GIT NHL 01/92," Journal of Clinical Oncology, Vol. 19, No. 18, 2001, pp. 3874-3878.

[42] Y. Lv, X. N. Wang and H. Liang, "Clinical Analysis of 200 Patients with Primary Gastric Lymphoma,” Zhonghua Wei Chang Wai Ke Za Zhi, Vol. 15, No. 2, 2012, pp. 157-160.

[43] F. Selçukbiricik, D. Tural, O. Elicin, S. Berk, et al., "Primary Gastric Lymphoma: Conservative Treatment Modality is Not Inferior to Surgery for Early-Stage Disease,” ISRN Oncology, Vol. 2012, 2012, p. 951816. doi:10.5402/2012/951816

[44] B. Coiffier, C. Gisselbrecht, A. Bosly, et al., "Ten Years Follow-Up of the GELA LNH98.5 Study, First Randomized Study Comparing R-CHOP to CHOP Chemotherapy in Patients with Diffuse Large B-Cell Lymphoma,” ASH 2009.

[45] A. Ruskoné-Fourmestraux, A. Lavergne, P. H. Aegerter, F. Megraud, et al., "Predictive Factors for Regression of Gastric MALT Lymphoma after Anti-Helicobacter Pylori Treatment,” Gut, Vol. 48, No. 3, 2001, pp. 297-303. doi:10.1136/gut.48.3.297

[46] L. T. Chen, J. T. Lin, J. J. Tai, G. H. Chen, et al., "Long-Term Results of Anti-Helicobacter Pylori Therapy in Early-Stage Gastric High-Grade Transformed MALT Lymphoma," Journal of the National Cancer Institute, Vol. 97, No. 18, 2005, pp. 1345-1353. doi:10.1093/jnci/dji277
[47] S. Nakamura, T. Matsumoto, H. Suekane, M. Takeshita, et al., "Predictive Value of Endoscopic Ultrasonography for Regression of Gastric Low Grade and High Grade MALT Lymphomas after Eradication of Helicobacter Pylori,” Gut, Vol. 48, No. 4, 2001, pp. 454-460. doi:10.1136/gut.48.4.454

[48] W. W. Ng, C. P. Lam, W. K. Chau, A. Fen-Yau Li, et al., "Regression of High-Grade Gastric Mucosa-Associated Lymphoid Tissue Lymphoma with Helicobacter Pylori after Triple Antibiotic Therapy," Gastrointestinal Endoscopy, Vol. 51, No. 1, 2000, pp. 93-96. doi:10.1016/S0016-5107(00)70399-3

[49] L. Cavanna, R. Pagani, P. Seghini, et al., "High Grade B-Cell Gastric Lymphoma with Complete Pathologic Remission after Eradication of Helicobacter Pylori Infection: Report of a Case and Review of the Literature," World Journal of Surgical Oncology, Vol. 6, 2008, p. 35.

[50] B. Cheson and M. Rummel Bendamustine, "Rebirth of an Old Drug,” Journal of Clinical Oncology, 2009, pp. 1492-1501.

[51] M. Rummel, E. Sahah, Al-Batran et al., "Bedamustine plus Rituximab is Effective as Favorable Toxicity Profile in the Treatment of Mantle Cell and Low Grade NonHodgkin's Lymphoma,” Journal of Clinical Oncology, Vol. 23, No. 15, 2005, pp. 3383-3389.

[52] K. Dunleavy, S. Pittluga, M. S. Czuczman, et al., "Differential Efficacy of Bortezomib plus Chemotherapy within Molecular Subtype of Diffuse Large B Cell Lymphoma,” Blood, Vol. 113, No. 24, 2009, pp. 6069-6076.

[53] F. J. Hernandez-Ilizaliturri, G. Deeb, P. L. Zinzani, et al., "Higher Response to Lenalidomide in Relapsed/Refractory Diffuse Large B Cell Lymphoma in Non-Germinal Center B-Cell-Like Than in Germinal Center B-Cell-Like Phenotype,” Cancer, Vol. 117, No. 22, 2011, pp. 50585066.

\footnotetext{
Abbreviation

PGL: primary gastric lymphoma,

MALT: mucosa associated lymphoepithelial tumor,

H. pylori: Helicobacter pylori,

DLBCL: diffuse large B-cell lymphoma,

R: rituximab
} 